\title{
Intervenciones para optimizar la oferta de métodos modernos de planificación familiar en la región tropical de Loreto, Perú*
}

\section{Interventions to Optimize the Modern Family Planning Method Supply in the Loreto Tropical Region, Peru}

\section{Intervenções para otimizar a oferta a métodos modernos de planejamento familiar na região tropical de Loreto, Peru}

Fecha de recepción: 25-11-15 Fecha de aceptación: 24-02-16 Disponible en línea: 03-05-16 doi:10.11144/Javeriana.rgyps15-30.ioom

Cómo citar este artículo:

Carmona G, Aspilcueta J, Cárdenas U, Flores S, Álvarez E, Solary L. Intervenciones para optimizar la oferta de métodos modernos de planificación familiar en la región tropical de Loreto, Perú. Rev. Gerenc. Polit. Salud. 2016; 15(30): 142-158. http://dx.doi.org/10.11144/Javeriana.rgyps15-30.ioom

\author{
Gloria Carmona** \\ Juan Aspilcueta*** \\ Ulalia Cárdenas $* * * *$ \\ Sara Flores $* * * * *$ \\ Elizabeth Álvarez****** \\ Lely Solary $* * * * * *$
}

Artículo de investigación. Proyecto financiado por el Instituto Nacional de Salud del Perú.

** Ms. Science, licenciada en Obstetricia, investigadora. Unidad de Análisis y Generación de Evidencias en Salud Pública, Instituto Nacional de Salud, Lima, Perú. Autor principal y autor corresponsal. Domicilio: Calle Santa Felicidad, Urbanización Pando, Tercera Etapa, Cercado de Lima. Correo electrónico: gcarmona@ins.gob.pe -gloriacarmona.c@ gmail.com.

**** MD. Ginecólogo. Ministerio de Salud. Coordinador nacional de la Estrategia de Salud Sexual y Reproductiva. Dirección General de Salud de las Personas, Ministerio de Salud.

***** Mg en Salud Pública. Licenciada en Obstetricia. Ministerio de Salud, Equipo Técnico de la Estrategia de Salud Sexual y Reproductiva. Dirección General de Salud de las Personas.

****** Licenciada en Obstetricia. Coordinadora regional de Salud Sexual y Reproductiva. Dirección Regional de Salud de Loreto, Perú.

$12^{* * * * * * * *}$ Ph. D. (c). MD. Mc Master University, Health Policy Program.

$12^{* * * * * * * *}$ Ph. D. (c). MD. Gerente del Instituto de la Dirección de Investigación, Instituto de Evaluación de Tecnología Sanitaria e Investigación, Essalud. 


\section{Resumen}

El uso de métodos de planificación familiar, principalmente de métodos anticonceptivos modernos - por su mayor eficacia-, está asociado a la disminución de casos de embarazo no deseado, abortos, y a una disminución de las tasas de fecundidad y mortalidad materna, viabilizando así una maternidad voluntaria y saludable. Sin embargo, su uso en el Perú es aún insuficiente, sobre todo en un departamento como Loreto que presenta el mayor porcentaje de demanda insatisfecha de planificación familiar a nivel nacional $(14,2 \%)$ y altas tasas de fecundidad $(3,8)$. Este es un problema importante para la salud sexual y reproductiva, y en general para la salud pública, ya que tiene repercusiones socioeconómicas relevantes. En este artículo se realiza una revisión narrativa de la evidencia disponible sobre tres intervenciones destinadas a incrementar la oferta de los métodos anticonceptivos modernos, su contextualización local y las consideraciones para su potencial implementación local.

Palabras clave: política de salud; sistemas de salud; planificación familiar; salud sexual y reproductiva

\section{Abstract}

The use of family planning methods, mainly modern contraceptive methods - due to their higher effectiveness - is associated with a reduction in the number of unplanned pregnancy and abortion cases, and also with a reduction in the maternal fertility and morbidity rates, leading to a greater visibility of a voluntary and healthy maternity. However, the use of these methods is still insufficient in Peru, especially in states such as Loreto, which has the highest unfulfilled demand rate for family planning methods on the national level (14.2\%) and high fertility rates (3.8). This is an important issue for sexual and reproductive health and, in general terms, for public health, as it has relevant social and economic results. In this article, we carry out a narrative review of the available evidence on three interventions aimed to increase the supply of modern contraceptive methods, their local contextualization, and considerations for potential local implementation.

Keywords: health policy; health system; family planning; sexual and reproductive health

\section{Resumo}

O uso de métodos de planejamento familiar, principalmente de métodos contraceptivos modernos — pela sua maior eficácia—, é associado à diminuição de casos de gravidez não desejada, abortos, e diminuição das taxas de fecundidade e mortalidade materna, possibilitando assim uma maternidade voluntaria e saudável. No entanto, o uso no Peru ainda é insuficiente, sobretudo em um departamento como Loreto que apresenta a maior porcentagem de demanda insatisfeita de planejamento familiar no nível nacional $(14,2 \%)$ e altas taxas de fecundidade $(3,8)$. Este é um problema importante de saúde sexual e reprodutiva e em geral para a saúde pública, pois tem repercussões socioeconómicas relevantes. Neste artigo realiza-se uma revisão narrativa da evidencia disponível sobre três intervenções destinadas a incrementar a oferta dos métodos anticoncepcionais modernos, sua contextualização local e as considerações para sua potencial implementação local.

Palavras-chave: política de saúde; sistemas de saúde; planejamento familiar; saúde sexual e reprodutiva 


\section{Introducción}

El Estado peruano reconoce el derecho de sus ciudadanos a decidir cuándo y cuántos hijos tener, a través del Servicio Nacional Gratuito de Planificación Familiar. La planificación familiar no solo está asociada a la disminución de casos de embarazo no deseado y abortos, sino también a menores tasas de fecundidad y de mortalidad materna y mejora del acceso a la educación infantil. Asimismo, fomenta el empoderamiento de las mujeres y reduce la pobreza, propiciando el desarrollo económico (1,2). Dentro del Servicio de Planificación Familiar se busca obtener una población informada en salud sexual y reproductiva y con acceso a métodos anticonceptivos tradicionales o modernos, con las diferencias correspondientes entre los dos grupos, ya que los métodos tradicionales presentan importantes tasas de falla, a causa de la información limitada en las usuarias sobre el periodo fértil del ciclo menstrual, mientras que los métodos modernos presentan mayor eficacia. El actual desafío del Servicio es incrementar el acceso a los métodos anticonceptivos modernos, el cual todavía es limitado a nivel regional, principalmente en departamentos de la selva como Loreto, que es la segunda región, luego de Lima, que reporta mayor número de muerte materna -32 por cada 100000 nacidos vivos-, menor uso de métodos anticonceptivos modernos y mayor porcentaje de demanda insatisfecha de planificación familiar: $14,2 \%$ versus $7,9 \%$ en Lima (3).

En Loreto, el uso de métodos anticonceptivos modernos ha caído de $49 \%$ en el 2013 a 43,3\% en el 2014 (4). En relación con la fecundidad, en esta región han sido pocos los avances. En el 2007, el número de hijos por mujer fue de 4,6, en tanto que en el 2013 fue de 3,8. Sin embargo, a nivel nacional, el número de hijos por mujer 144 se mantiene en $2,4(3,4)$. Estos indicadores reflejan que Loreto es un departamento donde el proceso de modernización de la sociedad y su influencia en el comportamiento hacia la fecundidad se encuentra relegado en relación con la capital (figura 1).
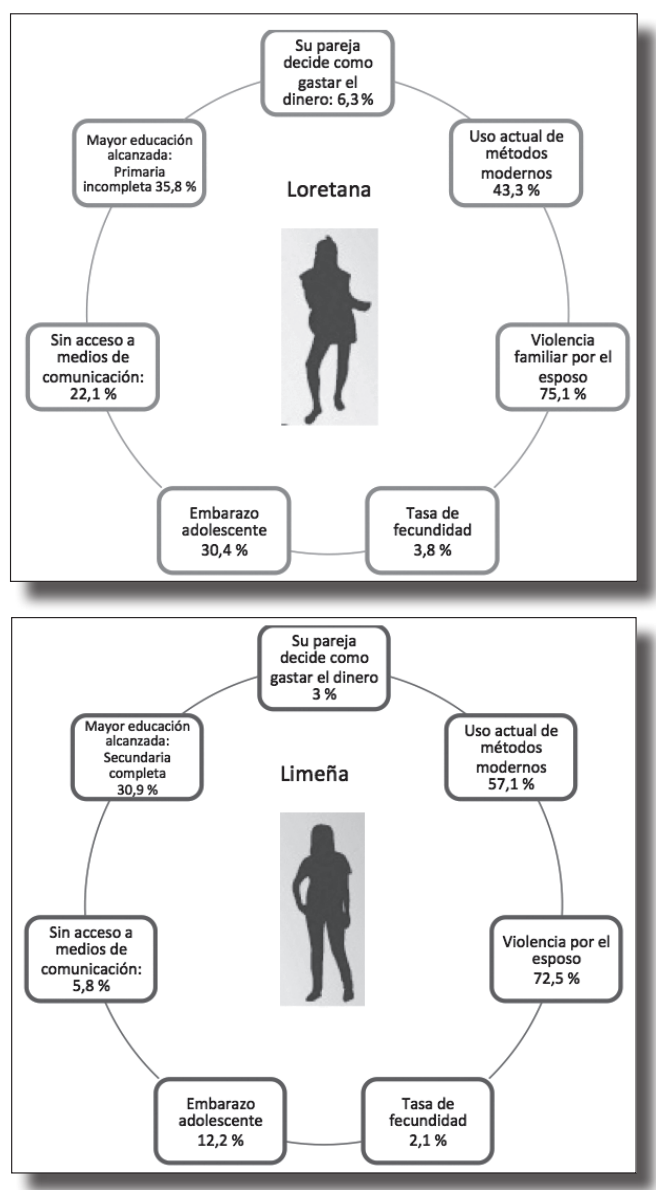

Figura 1. Situacion comparativa de aspectos DE LA SALUD SEXUAL Y REPRODUCTIVA EN LA MUJER LORETANA Y EN LA MUJER LIMEÑA.

Fuente: Endes 2014

Para comprender esta situación, es necesario tener en cuenta la diversidad de los contextos interculturales y geográficos de Loreto. Es asî cómo, habiéndose demostrado que el nivel de educación está positivamente asociado al uso de la planificación familiar, en Loreto existe una importante deserción escolar femenina $(26,2 \%)$ y una altísima tasa de ausencia de escolaridad femenina $(23,2 \%)(5)$. 
Este contexto es aún más complejo si se considera el inicio temprano de las relaciones sexuales, las altas tasas de violencia y el limitado poder de las mujeres para decidir sobre su cuerpo y sexualidad, lo que podría disminuir su capacidad para controlar aspectos de la vida como la fecundidad (6,7). En relación con las condiciones de los centros de salud en Loreto, diversos reportes resaltan la oferta limitada tanto de los métodos anticonceptivos modernos como de los demás servicios e insumos de planificación familiar, sea por abastecimiento insuficiente, por falta de personal de salud en horarios requeridos por la población, por dificultades en los transportes fluviales requeridos ocasionadas por ausencia de rutas comerciales o por combustible insuficiente para la distribución, entre otros (8-12).

En respuesta a esta situación, a la fecha se han implementado diversas estrategias que, potencialmente, podrían desarrollarse para mejorar la oferta de los servicios de planificación familiar, en particular de los métodos anticonceptivos modernos $(1,13)$. Tomando como base una de las últimas guías globales de la Organización Mundial de la Salud (OMs) basadas en la evidencia, este artículo tiene por objetivo revisar la evidencia vinculada con las intervenciones orientadas al fortalecimiento del acceso a los servicios de planificación familiar —con un énfasis en los métodos anticonceptivos modernos-y sus potenciales aplicaciones en Loreto (14).

\section{Método}

\section{Tipo de estudio}

Ante la necesidad de información sobre intervenciones que puedan optimizar el acceso y uso de métodos anticonceptivos modernos en localidades como Loreto, se realizó una revisión bibliográfica. Esta representa el soporte del problema de estudio, de donde se rescata la calidad y la profundidad en relación con los aportes y tendencias en la optimización de la oferta de dichos métodos.

\section{Fuentes de información}

De acuerdo con la estrategia de búsqueda de información, se revisaron diversas fuentes, entre ellas la guía global de la oms sobre salud materna y neonatal, se analizaron las recomendaciones en relación con el componente de planificación familiar (15). Se incluyó una búsqueda bibliográfica de artículos publicados entre los años 2005 y mayo del 2015 en la base de datos de la librería Cochrane y de evidencias internacionales publicadas en PubMed y Lilacs. Para esta búsqueda se establecieron los siguientes descriptores en inglés y en español: Family planning, maternal and child health, maternal and child care, contraception, birth control, primary health care, improvement, implementation, adherence, Choice, interventions, accessibility of health services, acceptability, availability of health services, access to health care, delivery of health care, developing countries, Latin America, Peru.

Al conjunto de estas evidencias internacionales y locales se adicionó la revisión de documentos nacionales y locales situacionales, regulatorios y normativos de planificación familiar. Finalmente, se incluyeron las referencias cruzadas encontradas.

De forma paralela, para la redacción del manuscrito se utilizó como referencia el manual elaborado por la oms en relación con el proceso de contextualización de las evidencias internacionales y nacionales encontradas acerca del contexto específico situacional de Loreto (14).

\section{Proceso de análisis}


Se realizó la lectura detallada de cada documento seleccionado, con el fin de analizar e interpretar, extrayendo temas, tendencias y contradicciones. Esto permitió identificar unidades de registro y análisis (16) que se agruparon por categorías tales como: intervenciones extramurales, intervenciones intramurales ampliadas, intervenciones de monitoreo y vigilancia. Estas categorías giraron alrededor de: usuarias y usuarios actuales y potenciales de planificación familiar, personal de salud implicado con las atenciones de planificación familiar y sistema de salud (figura 2).

De manera paralela a todo el proceso, se trabajó en coordinación con las principales direcciones del Ministerio de Salud, tanto centrales (Lima) como regionales (Loreto), a fin del análisis, la discusión y la contextualización de las evidencias científicas encontradas y su potencial desarrollo, considerando las intervenciones realizadas y sus principales regulaciones (17).

Este proceso empleó como guía el manual de la oms que incluye, entre otros aspectos, clarificar el problema, formular las opciones, identificar las consideraciones potenciales de implementación, el sistema de salud peruano actual, el sistema político y las necesidades de monitoreo y evaluación en el desarrollo potencial de las intervenciones encontradas y basadas en la evidencia científica (14). Este reporte no recomienda una u otra intervención; sin embargo, resalta la evidencia que existe en el abordaje del problema planteado y la necesidad de su contextualización en la comunidad objetivo.

\section{Resultados}

\section{Respuesta al problema}

En busca de optimizar el uso de recursos, orientar las decisiones y consolidar las in146 tervenciones que persigue este programa materno-neonatal, surge el Plan para la
Reducción de la Mortalidad Materna Perinatal, dentro del cual, uno de los objetivos sanitarios nacionales del 2007 hasta el 2020 es ampliar el acceso a la planificación familiar, reduciendo la demanda insatisfecha en poblaciones vulnerables (13). Según este plan, con el objetivo de garantizar la capacidad de respuesta del sector de salud, los establecimientos de salud deben cumplir con más del $80 \%$ de su capacidad resolutiva en la atención materno-perinatal. Para monitorizar este cumplimiento, la Encuesta Nacional a los Establecimientos de Salud (Enesa) 2012 evaluó la capacidad resolutiva de los establecimientos de salud con funciones obstétrico-neonatales básicas, esenciales e intensivas $(9,18)$.

La Dirección Nacional de Salud Sexual y Reproductiva ha elaborado normas, entre estas la Norma Técnica de Planificación Familiar, vigente desde el 2005, que incluye los procedimientos técnico-administrativos, los insumos anticonceptivos, las disposiciones, competencias de proveedores y las líneas de acción para la atención en planificación familiar, a fin de contribuir al logro de los objetivos reproductivos de los peruanos, basados en el respeto de los derechos humanos y la equidad de género (19). Esta norma técnica especifica las contribuciones actuales y potenciales del equipo de salud en estas actividades, después del entrenamiento en provisión de anticonceptivos (tabla 1).

En busca de fortalecer el conocimiento y el abordaje de las necesidades de la población con respecto a la planificación familiar, la norma técnica vigente en esta materia se complementa con distintos manuales, incluyendo aspectos de distribución de insumos, adecuación cultural y oyc, los cuales tienen aplicación a nivel nacional (18-20).

El presupuesto y la financiación para asegurar la información y el acceso a la planifi- 
Tabla 1. Competencias específicas de proveedores de salud según la Norma Técnica de Planificación Familiar. Ministerio de Salud

\begin{tabular}{|c|c|c|c|c|c|c|c|c|c|c|c|}
\hline \multirow[b]{2}{*}{$\begin{array}{l}\text { Personal de } \\
\text { la salud }\end{array}$} & \multicolumn{11}{|c|}{ Métodos anticonceptivos } \\
\hline & 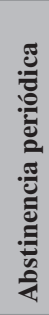 & 牙 & ن̃ & 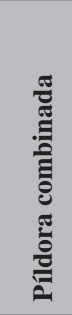 & 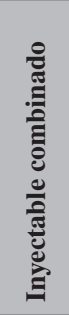 & 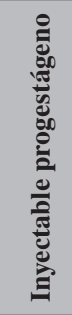 & 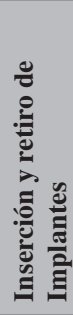 & 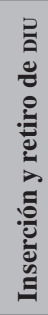 & 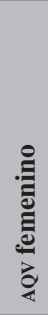 & 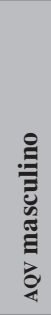 & 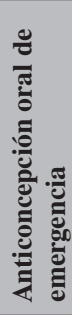 \\
\hline $\begin{array}{l}\text { Médico/a } \\
\text { ginecobstetra }\end{array}$ & $\mathrm{X}$ & $\mathrm{X}$ & $\mathrm{X}$ & $\mathrm{X}$ & $\mathrm{X}$ & $\mathrm{X}$ & $\mathrm{X}$ & $\mathrm{X}$ & $\mathrm{X}$ & $\mathrm{X}$ & $\mathrm{X}$ \\
\hline $\begin{array}{l}\text { Médico/a } \\
\text { general }\end{array}$ & $\mathrm{X}$ & $\mathrm{X}$ & $\mathrm{X}$ & $\mathrm{X}$ & $\mathrm{X}$ & $\mathrm{X}$ & $\mathrm{X}$ & $\mathrm{X}$ & $\mathrm{X}^{*}$ & $X^{*}$ & $\mathrm{X}$ \\
\hline Obstetra & $\mathrm{X}$ & $\mathrm{X}$ & $\mathrm{X}$ & $\mathrm{X}$ & $\mathrm{X}$ & $\mathrm{X}$ & $\mathrm{X}(2)$ & $\mathrm{X}$ & & & $\mathrm{X}$ \\
\hline $\begin{array}{l}\text { Enfermero/a } \\
\text { (capacitado) }\end{array}$ & $\mathrm{X}$ & $\mathrm{X}$ & $\mathrm{X}$ & $X(3)$ & $X(3)$ & $X(3)$ & & & & & $X(3)$ \\
\hline $\begin{array}{l}\text { Técnico/a en } \\
\text { enfermería } \\
\text { (capacitado) }\end{array}$ & $X$ & $X$ & $X$ & $X(3)$ & $X(3)$ & & & & & & \\
\hline
\end{tabular}

(2) Siempre y cuando esté calificado para ello.

(3) Proveerá el insumo e identificará signos de alerta. La prescripción la debe realizar el profesional autorizado. Fuente: Norma Técnica de Planificación Familiar, Ministerio de Salud, 2005

cación familiar provienen en la mayor parte del Ministerio de Economía y Finanzas, a nivel central, regional y local, a través del Presupuesto por resultado del Programa Presupuestal Materno-Neonatal (21,22). Es necesario mencionar que el presupuesto asignado a este programa y a su componente de planificación familiar ha ido incrementándose progresivamente en los últimos años (12,23). El Ministerio de Economía y Finanzas monitorea estos programas a través de diversos indicadores de resultados, evaluados en las encuestas nacionales, que dan información sobre el estado de la población y de la capacidad resolutiva de los centros de salud. En Loreto, la mayor parte del presupuesto regional asignado a salud sexual y reproductiva se destina a los contratos del profesional de salud, en tanto que el presupuesto restante se asigna a otras actividades como, por ejemplo, actividades de supervisión, indumentaria, asistencias técnicas, impresión y elaboración de distintos tipos de formatos y material de escritorio (12).

Factores relacionados con las intervenciones actuales y la oferta en Loreto que contribuyen a la inequidad al presente problema

Identificar los factores de riesgo asociados al uso insuficiente de métodos anticonceptivos modernos viabiliza una aproximación a las potenciales intervenciones que se podrían desarrollar para abordar este problema.

El perfil de las personas con demanda insatisfecha de planificación familiar en Loreto se caracteriza por una baja escolaridad, alta ruralidad, difícil acceso geográfico, vulnerabilidad en relación con la inequidad en género, dificultades de empoderamiento sobre la toma de decisiones de su cuerpo y otras cuestiones, además de dependencia económica (1). Esta situación se torna aún más 
compleja con factores como la adolescencia, los proyectos de vida tradicionales - que resaltan el rol reproductivo, direccionándolo a la maternidad o a la paternidad tempranay los vacíos de información en relación con la planificación familiar y la fisiología del cuerpo (24). En Loreto, la gran violencia basada en género implica que las mujeres no acudan al establecimiento de salud, ya que no cuentan con la autorización de su pareja para usar un anticonceptivo. Sin embargo, algunas mujeres optan por usarlo a escondidas, corriendo el riesgo de ser maltratadas y ser sospechosas de infidelidad en caso de que sean descubiertas (25).

En relación con la basta población nativa y la diversidad étnica de Loreto, no se tiene referencia sobre el uso de anticonceptivos y, según la experiencia de la Dirección Nacional de Salud Sexual y Reproductiva, son las mujeres y los apus ${ }^{1}$ quienes más se resisten a ser atendidos por profesionales de salud, al punto de afirmar que así se atenta contra su grupo étnico (25). Esta población se encuentra en las zonas más alejadas de Loreto, sin rutas comerciales fluviales. Estas zonas están conformadas, principalmente, por una población muy dispersa de tres habitantes por kilómetro cuadrado, radio en el cual se cuenta, en general, con un técnico en enfermería y un promotor de salud (12).

Respecto a los servicios, el desconocimiento sobre la disponibilidad de atención en planificación familiar durante las tardes, el pensar que no habrá personal femenino ni horarios compatibles con los de la población, se han expresado por parte de las usuarias como unas de las dificultades en el acceso a los servicios de planificación familiar (26).

El abastecimiento oportuno y suficiente de insumos anticonceptivos en los centros

1 Líderes comunitarios. de salud, varias ocasiones se reduce a preservativos e inyectables trimestrales. La disponibilidad de estos insumos — píldoras e inyectables mensuales, píldoras de emergencia, dispositivos intradérmicos e intrauterinos y preservativo femenino- está sujeta a la agilidad de los procesos de adquisición o distribución, con su consecuente disponibilidad de insumos, la accesibilidad geográfica, la existencia de rutas comerciales y el abastecimiento suficiente de combustible para los traslados. De esta manera, se incrementan estos sistemas de distribución. Durante los traslados para la entrega de sus informes o referencias, el personal de salud también busca abastecer a los centros de salud de métodos anticonceptivos modernos $(16,17)$. La deficiencia de alguno de estos sistemas se traduce en un desabastecimiento de estos métodos por periodos considerables en los establecimientos de salud (12).

En los sistemas de salud de países desarrollados existe una oferta de alrededor de veinte tipos de métodos anticonceptivos modernos, los cuales se ajustan de acuerdo a las necesidades anticonceptivas y características de las usuarias $(18,19,9)$. En el Perú esta oferta es reducida y, a pesar de que se ha venido implementando la distribución de nuevos métodos anticonceptivos modernos, solo algunos de ellos están disponibles en la práctica, en tanto que hay desabastecimiento por periodos considerables. Por ejemplo, en Loreto, diversas usuarias priorizan la importancia de contar con su ciclo menstrual de manera permanente y, sin embargo, la ampolla trimestral es el único insumo disponible que puede ser controlado por ellas mismas en el momento de la consulta $(8,12,14,16,20)$.

En relación con recursos humanos, Loreto es el departamento con menor falta de profesionales de salud, y la mayor parte de atenciones profesionales en planificación familiar se da en los 44 centros de salud I-3, con un horario 
de atención de 7 a.m. a 1 p.m. Estos cuentan además con por los menos un profesional de obstetricia y un técnico de enfermería o promotor de salud en los servicios de planificación familiar. En este sentido, uno de los factores asociados a la demanda insatisfecha es que muchas mujeres no encuentran citas dentro de estos horarios y el que no haya atención durante las tardes $(12,21)$. De los establecimientos de salud I-3 ubicados en las siete provincias que conforman el departamento de Loreto, en tres provincias estos funcionan solo durante las mañanas. Sin embargo, en los cuatro restantes se encuentra un profesional en obstetricia tanto en la mañana como en las tardes, sin que ello implique la oferta ampliada del consultorio de planificación familiar. Según la directiva de guardia comunitaria, este personal de salud debe realizar labor extramural durante las tardes o labor en otros espacios intramurales obstétricos, como emergencia. Por otro lado, también se reconoce una insuficiente cobertura y realización tanto de actividades extramurales como de una oyc suficiente e oportuna $(25,27)$.

Por otro lado, las actividades de promoción de salud incluyen la difusión de información sobre planificación familiar. Estas actividades hacen la diferencia entre el conocer un método solo por su nombre o tener una idea real de cómo se emplea y sus implicancias, otorgando un buen nivel de información. Sobre este aspecto los reportes nacionales sostienen que dichas actividades han sido insuficientes en distintos centros de salud evaluados en Loreto (28). Cabe resaltar que para el desarrollo de estas actividades es fundamental que los establecimientos de salud cuenten con la disponibilidad de los documentos técnicos relacionados, sin embargo, entre el 2011 y el 2012 el 50\% de los centros de salud participantes no contaba con diversos documentos técnicos relacionados (9).
Finalmente, en Loreto las capacitaciones al personal profesional sobre metodología anticonceptiva se realizan de forma anual, con facilitadores enviados desde Lima (25). Sin embargo, los técnicos de enfermería muchas veces quedan relegados. En la práctica, no hay evidencia de que los técnicos o promotores de salud hayan sido capacitados en la provisión de anticonceptivos, que se hayan realizado réplicas en las zonas periféricas o que se traslade al personal técnico o comunitario para su participación $(17,21)$. Esta situación imposibilita al personal técnico o auxiliar ofrecer algún servicio de planificación familiar, según la Norma Técnica de Planificación Familiar, vigente desde el 2005.

Para mejorar la oferta en los servicios de planificación familiar, considerando la actual demanda insatisfecha, el personal de salud sexual y reproductiva representa un nexo entre los usuarios y el sistema de salud. De acuerdo con ello, la guía global de la oms considera fundamental a este equipo de salud en el fortalecimiento de las actividades de difusión, supervisión y monitoreo de la planificación familiar, dirigiendo sus prácticas hacia la ampliación de las actividades en la comunidad $(14,15)$.

\section{Intervenciones para abordar el problema}

\section{Optimización de la referencia domiciliar y} mapeo de la jurisdicción asignada a través de la oyc. La literatura científica sostiene que dentro de los desafíos para el fortalecimiento de las actividades de planificación familiar se encuentra la necesidad de asegurar la sostenibilidad de los servicios y los insumos, optimizando la calidad de la información, de forma tal que permita disipar conceptos erróneos sobre la anticoncepción (29). En relación con ello, una reciente revisión Cochrane de Lewin del año 2012 concluye que

2 Ver tablas 2 y 3. 


\begin{tabular}{|c|c|c|c|c|}
\hline 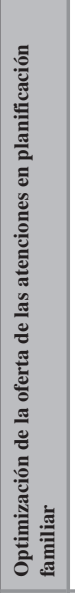 & 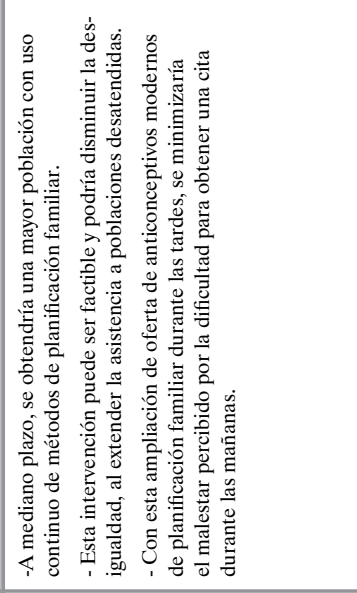 & & 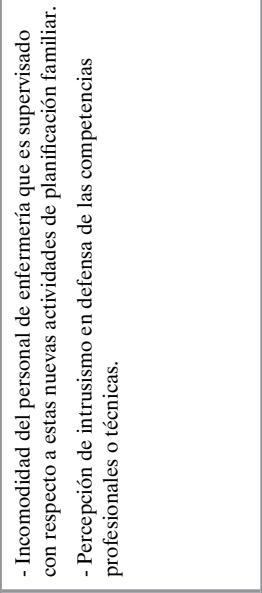 & 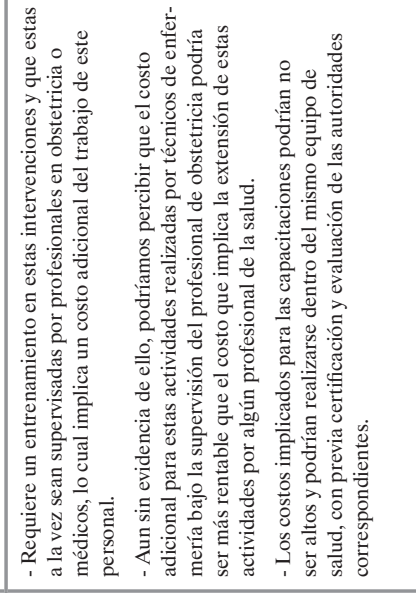 \\
\hline 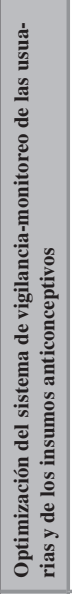 & 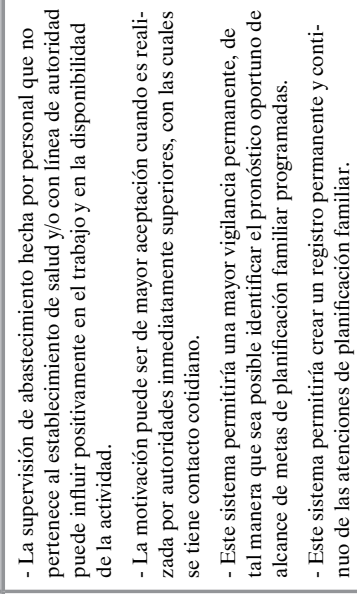 & 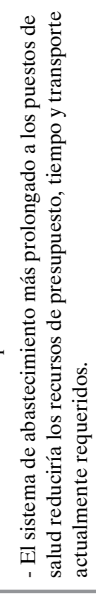 & 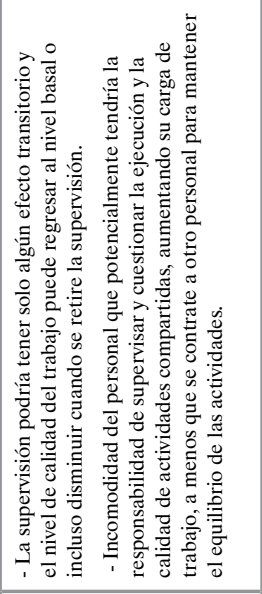 & 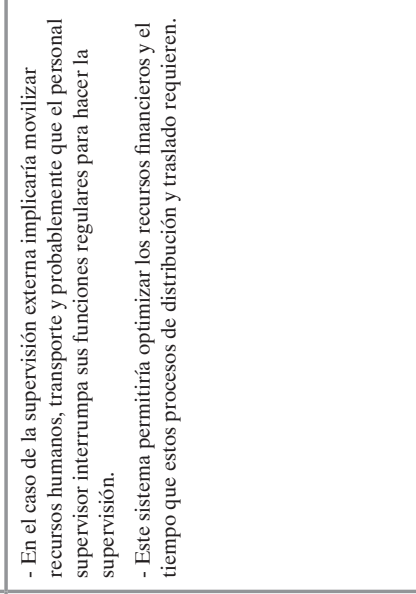 \\
\hline 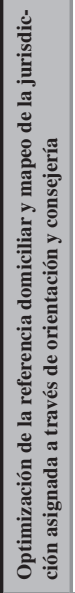 & 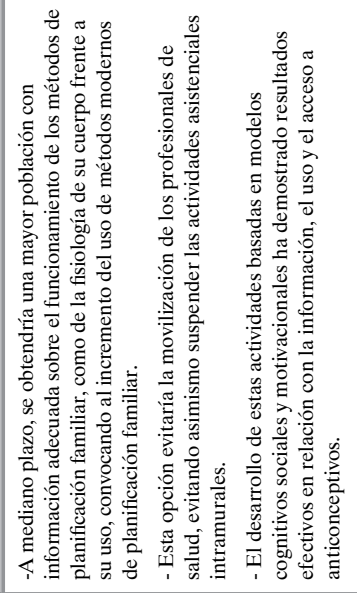 & 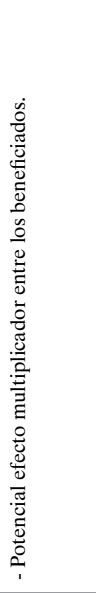 & 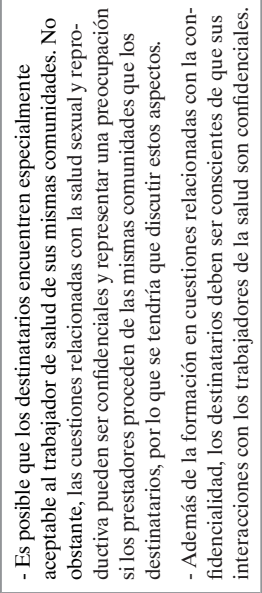 & 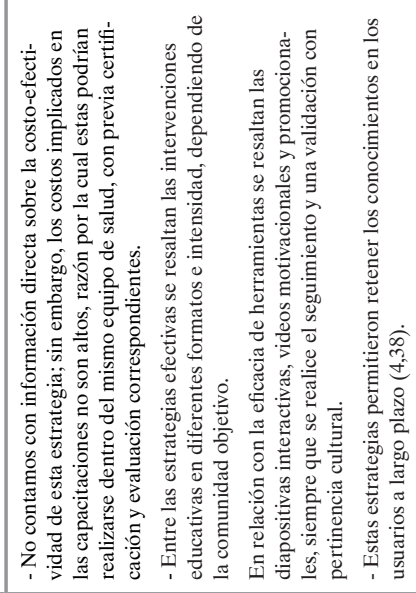 \\
\hline 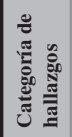 & & & 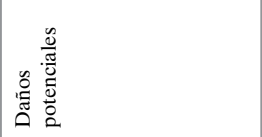 & 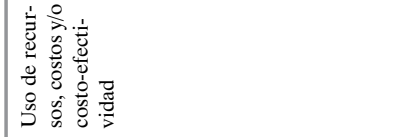 \\
\hline
\end{tabular}




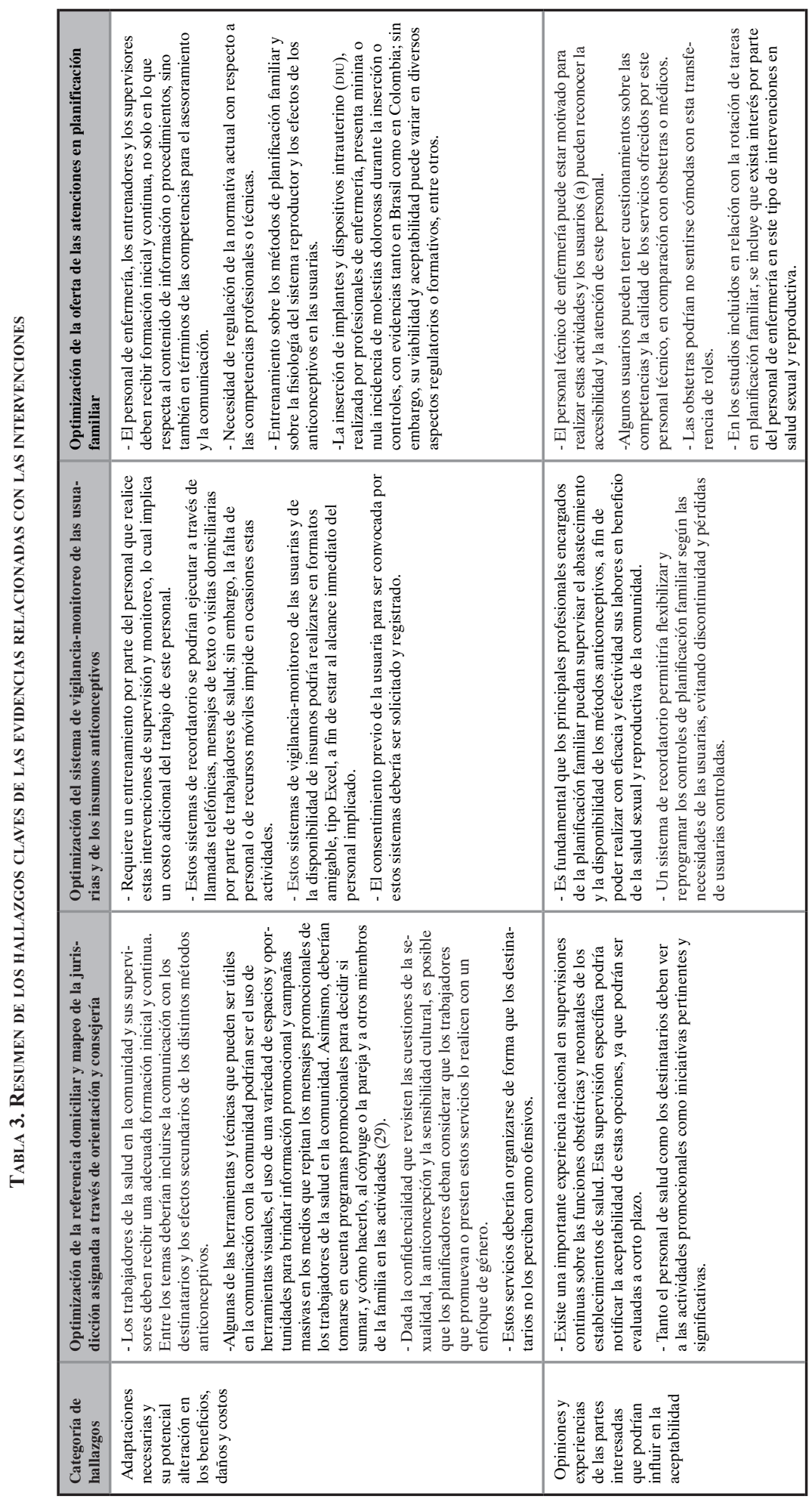


las sesiones extramurales de oyc realizadas por colaboradores de salud como los técnicos o agentes comunitarios, con capacitación previa sobre planificación familiar, permitiría fortalecer la promoción de comportamientos y servicios beneficiosos para la salud sexual y reproductiva, considerándola una opción eficaz, aceptable y factible que podría disminuir la desigualdad y extender los servicios a poblaciones desatendidas (30). Estas actividades extramurales incluyen el mapeo dentro de la jurisdicción asignada, a fin de identificar y referenciar a los usuarios continuadores y potenciales de planificación familiar. Una segunda revisión Cochrane en relación con la oYc y la referencia domiciliar en planificación familiar concluye que esta intervención tiene el potencial de disminuir la equidad, incrementando el acceso a la planificación familiar, sin problemas de seguridad asociados, ya que en diversos lugares los promotores de la salud ya administran asesoramiento relativo a planificación familiar $(23,31)$. Una tercera revisión Cochrane sostiene que los componentes educativos con múltiples sesiones son prometedores, siempre que se adapten a las locaciones que se desarrollen y se sujeten a evaluación $(25,32)$. Como ejemplo de la efectividad de estas intervenciones se puede citar una revisión Cochrane que concluye que las actividades de oyc realizadas por promotores comunitarios durante la etapa prenatal, y dirigidas a parejas durante el postparto, lograron un aumento significativo en el uso de métodos modernos de planificación familiar (33). Estas actividades permitirían disminuir las barreras de acceso geográfico, si el agente comunitario se acerca a la población. A su vez, la oyc diferenciada en género generaría oportunidades de empoderamiento en las mujeres, alternativas de manejo en relación con la violencia basada en género y escenarios de reflexión sobre la necesidad de la planificación familiar en los varones.
La OMS sugiere que se implementen estas intervenciones bajo supervisión y evaluación específica.

\section{Optimización del sistema de vigilancia- monitoreo de las usuarias y de los insumos} anticonceptivos. En relación con la prevención y recuperación de las usuarias de planificación familiar cuando no asisten a la cita programada de planificación familiar, a fin de reconvocarlas, se encontró una revisión sistemática Cochrane que evalúa los sistemas de recordatorios por teléfono, los cuales se definen como cualquier acción ejecutada para contactar a la usuaria poco antes de que le corresponda su próxima consulta (33).

Estos sistemas podrían fortalecerse a través de mensajes de texto, telefonía móvil o aplicativos móviles, y ello representaría una nueva alternativa de abordaje. La acción de identificación de estos usuarios podría ser realizada por el profesional, en coordinación con el personal técnico o auxiliar, a fin de evitar discontinuidad o pérdidas de usuarias o usuarios de planificación familiar, y programar las citas en horarios viables. En ese sentido, se incrementaría el conocimiento de la usuaria sobre la disponibilidad oportuna y flexible de atenciones en planificación familiar.

En relación con la falta de disponibilidad de suministros anticonceptivos, los desafíos se concentran en el fortalecimiento de las cadenas de suministro, la coordinación de distintos proveedores, las actividades de monitoreo y las actividades de supervisión, a fin de asegurar la disponibilidad permanente de la gama anticonceptiva ofrecida gratuitamente por el Estado (29). Una revisión sistemática de Cochrane concluye que la supervisión presenta efectos positivos en la práctica de los proveedores de salud, sin sugerir todavía algún tipo específico de supervisión (34). Considerando que estas actividades son complejas en su ejecución, se 
requeriría la supervisión del abastecimiento de los insumos de planificación familiar en los centros de salud, de tal manera que además de garantizarse su disponibilidad en el área adjudicada, se garantice su entrega, distribución y disponibilidad por mayor periodo de tiempo al personal de los puestos de salud más próximos y pertenecientes a las zonas periféricas más alejadas y sin rutas fluviales comerciales. Estas acciones podrían evitar el abastecimiento insuficiente de insumos anticonceptivos. Estos insumos de planificación familiar incluyen tanto anticonceptivos como material de difusión - rotafolios, volantes, etc.

Optimización de la oferta de las atenciones en planificación familiar. Dentro del desarrollo de nuevos sistemas de distribución de anticonceptivos modernos recomendados por la oms, y dirigidos a poblaciones con difícil acceso como Loreto, se describen las atenciones realizadas por el personal técnico y profesional de enfermería $(14,19)$. Una revisión Cochrane del 2014 concluye que el personal de enfermería, con un entrenamiento apropiado, puede realizar las actividades de planificación familiar con alta calidad. Esta revisión realizó un seguimiento de los beneficios de esta intervención por doce meses. Reconociendo que la aceptabilidad de estas intervenciones puede variar de acuerdo a diversos factores, en general podrían ser eficaces $(28,35)$. Con resultados similares, diversos estudios concluyen que estas intervenciones de administración de anticonceptivos hormonales y anticonceptivos de implantes podrían disminuir las inequidades de uso de métodos de planificación familiar en poblaciones desatendidas y con bajo acceso a los servicios de salud $(27,36,37)$. Considerando estas evidencias, la reciente Guía Global de la oms recomienda incrementar la oferta de provisión de anticonceptivos hormonales, en la inserción y extracción tanto de implantes anticonceptivos como de dispositivos intra- uterinos, a través de los profesionales de enfermería, con previa capacitación. A su vez, la oms expresa que estas recomendaciones están sujetas a las consideraciones para su implementación en cada contexto, en relación con distintos aspectos como viabilidad remunerativa, regulatoria y formativa de las competencias profesionales.

En ese sentido, incrementar la oferta de atenciones de planificación familiar, incluyendo la distribución de anticonceptivos — de barrera, inyectables y orales a usuarias continuadoras-, a través de la apertura del consultorio de planificación familiar durante las tardes en todos los centros de salud (I-3) que no cuenten previamente con el profesional para estas atenciones, podría implementarse a través del personal técnico de enfermería. Este sistema realizado por el personal técnico de enfermería y supervisado por el profesional autorizado de las prescripciones anticonceptivas, probablemente sea más viable en aspectos presupuestales, en comparación con la capacitación formativa, las exigencias regulatorias y las adecuaciones remunerativas que se requerirían en otro profesional de la salud. Estas actividades podrían realizarse con previa capacitación en diversos aspectos de planificación familiar. En relación con ello, una reciente revisión Cochrane concluye que el suministro de algunos anticonceptivos por parte de técnicos de enfermería o agentes comunitarios con capacitación previa sobre planificación familiar, permitiría fortalecer la promoción de comportamientos y servicios beneficiosos para la salud sexual y reproductiva, considerándola una opción eficaz, aceptable y factible que podría disminuir la desigualdad, extendiendo los servicios a poblaciones desatendidas $(38,39)$. Además, estas intervenciones permitirían a las usuarias encontrar una cita dentro de horarios más convenientes para ellas, coordinados con un personal de salud previamente entrenado sobre metodología anticonceptiva. 


\section{Discusión}

Los hallazgos de esta revisión resaltan tres intervenciones basadas en la evidencia, las cuales podrían implementarse para fortalecer la oferta de métodos anticonceptivos modernos en los establecimientos de salud de Loreto que cuenten con por lo menos con un profesional en obstetricia y un colaborador en los servicios de planificación familiar, sea técnico de enfermería o promotor de salud, y teniendo en cuenta los factores contextuales actuales de la población y del sistema de salud relacionados con la demanda insatisfecha en planificación familiar. La implementación de estas intervenciones requiere un conjunto de consideraciones contextuales, como los beneficios o daños potenciales para los implicados, el uso de recursos, los costos, las adaptaciones necesarias para el abordaje de los beneficios o daños potenciales, las barreras potenciales y las opiniones y experiencias de los implicados que puedan influir en la aceptabilidad de las intervenciones (tablas 2, 3 y 4). En ese sentido, los planificadores y tomadores de decisiones deben considerar diversas cuestiones relacionadas con la ampliación de las actividades de planificación familiar y la extensión de las responsabilidades del personal de salud que es necesario considerar en estas intervenciones. Estas tareas incluyen la distribución de las funciones entre los cuadros de personal, cuestiones regulatorias, participación de las partes interesadas, capacitación, supervisión, sistemas de derivación, cadenas de suministros y posibles cambios en las remuneraciones y demás incentivos. Además, la iniciación y el mantenimiento de anticonceptivos requerirían que hubiese un sistema de despensa y/o recambios de los anticonceptivos por parte del personal de salud considerado en estas intervenciones específicas.

La motivación hacia la necesidad local de 154 fortalecimiento del acceso a la planificación familiar debería ser parte de una interven- ción más amplia y focalizada - por ejemplo, la capacitación con enfoque comunitario considerando las características anticonceptivas locales - para que no sea vista solo como una actividad de designación o trasferencia de roles, sino como una estrategia diseñada para mejorar la demanda insatisfecha de métodos modernos de planificación familiar en la localidad. Por otro lado, algunos proveedores de salud pueden introducir sus propios criterios para seleccionar los destinatarios de sus servicios, como por ejemplo el estado civil y la edad. Para evitar estos juicios es necesario un entrenamiento integral en planificación familiar.

Otros factores que podrían influir en la efectividad de estas intervenciones son la falta de conocimiento sobre la fisiología y el efecto de cada anticonceptivo, las creencias religiosas, el apoyo del cónyuge, etc. Además, hay aspectos de la salud sexual y reproductiva en los que influyen de manera importante las creencias propias de las comunidades como, por ejemplo, hacia la anticoncepción y hacia la capacidad de la mujer para decidir sobre su propio cuerpo, por lo que se deben considerar los aspectos de pertinencia cultural con enfoque de género. Finalmente, aspectos como la información oportuna al personal de salud implicado, a través de las capacitaciones, y la difusión suficiente y oportuna de los servicios de planificación familiar a la población, son consideraciones que es preciso tener en cuenta.

Algunas de estas intervenciones son más prácticas de realizar, pero todas requieren considerar aspectos regulatorios, ampliación de responsabilidades, participación de las partes interesadas y considerar cambios en las remuneraciones y demás incentivos.

La organización y sustentación de estas intervenciones específicas y la contextualización del uso insuficiente de métodos anticoncep- 


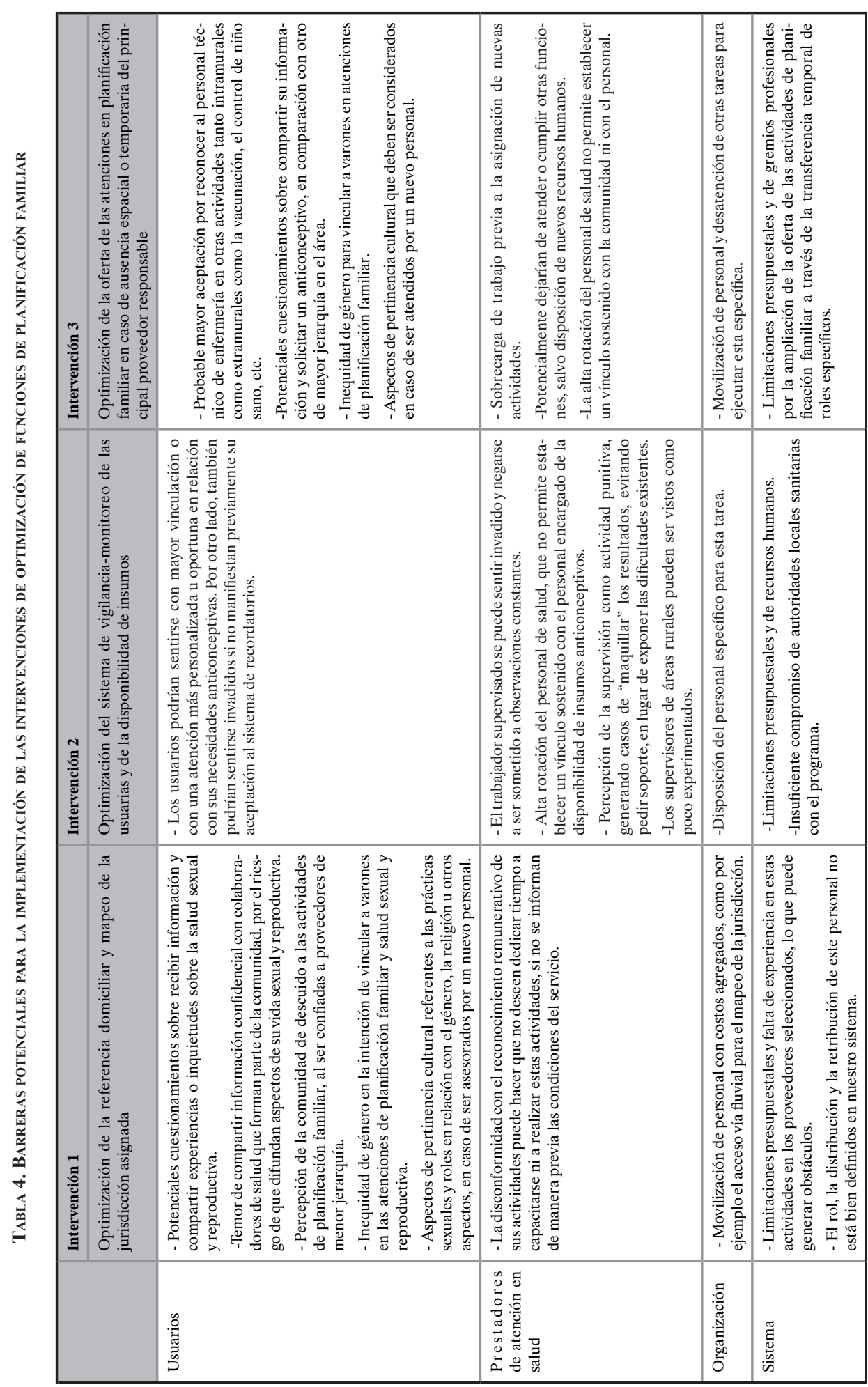


tivos modernos en Loreto, es producto de un trabajo articulado e intersectorial entre en Instituto Nacional de Salud y las direcciones de salud sexual-reproductiva centrales (Lima, Ministerio de Salud) y regionales (Dirección Regional de Salud de Loreto). Para que estas intervenciones logren ser consideradas políticas públicas regionales o incidir en los tomadores de decisiones, se requiere, entre otros aspectos, un diálogo deliberativo tanto con el personal técnico asistencial como con diversas entidades intersectoriales relacionadas con el tema de planificación familiar en Loreto, incluyendo a la sociedad civil, con el propósito de afinar su contextualización en Loreto y las consideraciones para su implementación, para que puedan traducirse en una política pública regional de salud sexual y reproductiva.

\section{Conclusiones}

Los hallazgos de esta revisión resaltan tres intervenciones basadas en evidencia, las cuales podrían implementarse para fortalecer la oferta de métodos anticonceptivos modernos en los establecimientos de salud de Loreto que cuenten con por lo menos un profesional en obstetricia y un colaborador en los servicios de planificación familiar, sea técnico de enfermería o promotor de salud. Estas intervenciones son: 1) las actividades de mapeo de la jurisdicción y referencia domiciliaria, a través de las actividades de orientación y consejería de los agentes comunitarios; 2) un sistema de monitoreo a través de mensajes de texto y/o visitas domiciliarias, tanto de insumos anticonceptivos como de la continuidad oportuna de las usuarias a través del personal técnico o auxiliar en planificación familiar; y 3) la ampliación de la oferta de las atenciones en planificación familiar a través de la designación de funciones a otro personal 156 de la salud en horarios extendidos, cuando el profesional encargado no se encuentre disponible. Finalmente, cabe expresar que la oferta de los servicios de planificación familiar va más allá de la sola disponibilidad de métodos anticonceptivos modernos. Conjuntamente, es necesario contar con personal de salud calificado que pueda brindar la oyc que le permita a la usuaria o usuario tomar una decisión asertiva y consciente de su elección anticonceptiva. En este artículo se han presentado varias intervenciones que podrían implementarse o complementar lo ya existente y se ha discutido la evidencia disponible sobre ellas.

\section{Agradecimientos}

Agradecemos al licenciado Henrry Daza por sus aportes a este manuscrito.

\section{Referencias bibliográficas}

1. Gutiérrez-Aguado A. Intervenciones del Programa Presupuestal por resultados en salud maternoneonatal. Rev Peru Med Exp Salud Pública. 2013; 30 (3): 524-5.

2. Centro de Investigación y Desarrollo del Instituto Nacional de Estadística e Informática. Uso de métodos anticonceptivos en las provincias del Perú. 2005 [Internet]. Disponible en: http://www.inei.gob. pe/media/MenuRecursivo/publicaciones_digitales/ Est/Lib0896/Libro.pdf.

3. Instituto Nacional de Estadística e Informática (INEI). Encuesta Demográfica y de Salud Familiar, 2014. Perú: Instituto Nacional de Estadística e Informática; 2015.

4. Instituto Nacional de Estadística e Informática (INEI). Encuesta Demográfica y de Salud Familiar, 2014, capítulo 4: Planificación familiar. Perú: Instituto Nacional de Estadística e Informática; 2015.

5. Instituto Nacional de Estadística e Informática (INEI). Encuesta Demográfica y de Salud Familiar 2012. Características generales de las mujeres [Internet]. Disponible en: http://proyectos.inei. gob.pe/endes/2012/.

6. Minaya León P. Situación del embarazo adolescente. Avances y desafíos en la salud sexual y reproductiva de las y los adolescentes [Internet]. Disponible en: http://www.mesadeconcertacion. org.pe/documentos/documentos/PRESENTACIONVICEMINISTRO-DE-SALUD.pdf.

7. Instituto Nacional de Estadística e Informática (INEI). Encuesta Demográfica y de Salud Familiar 2012. Violencia contra las mujeres, niñas y niños 
[Internet]. Disponible en: http://proyectos.inei. gob.pe/endes/2012/.

8. Instituto Nacional de Estadística e Informática (INEI). Cuadros estadísticos nacionales y departamentales. Resultados de los Establecimientos de Salud con Funciones Obstétricas y Neonatales Básicas (FONB). Loreto [Internet]. Disponible en: http://www.unfpa.org.pe/InfoEstadistica/2013/ EncuestaEsFoyN/index.html.

9. Instituto Nacional de Estadística e Informática (INEI). Encuesta a Establecimientos de Salud con Funciones Obstétricas y Neonatales. Enesa 20092012 [Internet]. Disponible en: http://www.unfpa. org.pe/InfoEstadistica/2013/EncuestaEsfoyN/ index.html.

10. Mesa de Concertación para la Lucha contra la Pobreza. Seguimiento concertado al programa presupuestal salud materno neonatal (SMN) [Internet]. Disponible en: http://www.mesadeconcertacion. org.pe/documentos/documentos/doc_01693.pdf.

11. Gobierno Regional de Loreto, Dirección Regional de Salud Loreto. Manual de organización y funciones; 2012.

12. Comunicación personal. Responsable de la Estrategia de Salud Sexual y Reproductiva. Diresa. Loreto - UNAGESP.CNSP. Instituto Nacional de Salud; 2015.

13. Plan estratégico nacional para la reducción de la mortalidad materna y perinatal. 2009-2015. Reporte RM.207-2009.

14. World Health Organization (wHO). Optimizing health worker roles to improve access to key maternal and newborn health interventions through task shifting. Contextualizing the guidelines - workbook [Internet]. Disponible en: http://www.optimizemnh. org/Annexes/Annex_8_Contextualizing_Workbook.pdf.

15. World Health Organization (wHo). Optimizing health worker roles for maternal and newborn health [Internet]. [acceso: septiembre $15 \mathrm{del}$ 2015]. Disponible en: http://www.who.int/reproductivehealth/publications/maternal_perinatal health/97892450 4843/en/.

16. Lavis JN, Røttingen J-A, Bosch-Capblanch X, Atun R, El-Jardali F, Gilson L, et al. Guidance for Evidence-Informed Policies about Health Systems: linking guidance development to policy development. PLoS Med. 2012 Mar 13; 9 (3): e1001186.

17. Lavis JN, Oxman AD, Lewin S, Fretheim A. Support tools for evidence-informed health policymaking (STP). Health Res Policy Syst. 2009 Dec 16; 7 (Suppl 1): I1.

18. Capacidad resolutiva para la atención materna y neonatal mejoró en región Apurímac [Internet] [acceso: 13 de mayo del 2014]. Disponible en: http://www.parsalud.gob.pe/publicaciones-institucionales/157-actividadesmenu/ capacidad-resolutiva-para-la-atencion-materna-yneonatal-mejoro-en-regionapurimac/920.

19. Ministerio de Salud. Norma técnica de Planificación Familiar. 2005.

20. Ministerio de Salud. Manual de Orientación y Consejería en Salud Sexual y Reproductiva. Reporte RM 290-2006.
21. Adecuación cultural de la orientación y consejería en salud sexual y reproductiva.

22. Ministerio de Salud. Directiva sanitaria para la distribución del condón masculino.

23. Ministerio de Economía y Finanzas - Dirección General del Presupuesto Público. Avances al 2011. Reporte de progreso en la obtención de resultados de los programas presupuestales iniciados en el 2008 [Internet]. Disponible en: http://www. mef.gob.pe/contenidos/presu_publ/ppr/reporte_progreso_1ersem2011.pdf.

24. Villacorta $\overline{\mathrm{M}}$. Comportamientos frente a la anticoncepción y su relación con algunas características individuales familiares de las mujeres actualmente unidas, en una Comunidad de la Selva Baja. Tesis de Maestría, UPCH.

25. Flores S. Comunicación personal. Coordinación Nacional Estrategia Sanitaria Nacional de Salud Sexual y Reproductiva. Dirección General de Salud de las Personas. Ministerio de Salud; 2015.

26. Instituto Nacional de Estadística e Informática [INEI]. Encuesta Demográfica y de Salud Familiar (Endes). Salud materna [Internet]. Disponible en: http://proyectos.inei.gob.pe/endes/2012/.

27. Normatividad de guardias hospitalarias y comunitarias [Internet] [acceso: 14 de septiembre del 2015]. Disponible en: https://docs.google.com/document/ d/1IsODKKHQnMbXdTeW1mFZ096b4w1WZz gOGAW1z D-JghE/edit?usp=embed_facebook.

28. Instituto Nacional de Estadística e Informática Endes. Indicadores: Objetivos de Desarrollo del Milenio [Internet]. Disponible en: http://proyectos. inei.gob.pe/endes/2012/.

29. d'Arcangues CM, Ba-Thike K, Say L. Expanding contraceptive choice in the developing world: lessons from the Lao People's Democratic Republic and the Republic of Zambia. Eur J Contracept Reprod Health Care Off J Eur Soc Contracept. 2013 Dec; 18 (6): 421-34.

30. Lewin S, Munabi-Babigumira S, Glenton C, Daniels $\mathrm{K}$, Bosch-Capblanch X, van Wyk BE, et al. Lay health workers in primary and community health care for maternal and child health and the management of infectious diseases. Cochrane Database Syst Rev. 2010; (3).

31. Ministerio de Economía y Finanzas. Estructuras programáticas y funcionales de los programas presupuestales del año fiscal 2014 [Internet]. Disponible en: http://www.mef.gob.pe/contenidos/presu publ/anexos/PP_Estructuras_2014_20130514.pdf.

32. Dirección Regional de Salud Loreto. Evaluación de la gestión presupuestal 2014 y ajuste de metas en el marco de la acción 8 del plan de articulación. Dirección Regional de Salud Loreto; 2015.

33. Halpern V, Lopez LM, Grimes DA, Gallo MF. Strategies to improve adherence and acceptability of hormonal methods of contraception. Cochrane Database Syst Rev. 2011; (4).

34. Bosch-Capblanch X, Liaqat S, Garner P. Managerial supervision to improve primary health care in low- and middle-income countries. Cochrane Database Syst Rev. 2011; (9). 
35. Gobierno Regional de Loreto. Infraestructura Sanitaria - Región Loreto. Dirección Regional de Salud Loreto; 2015.

36. Gutiérrez M. La planificación familiar como herramienta básica para el desarrollo. Rev Peru Med Exp Salud Pública. 2013 Jul; 30 (3): 465-70.

37. Dirección Regional de Salud de Loreto. Acciones del 2013.

38. Glenton C. A systematic review of barriers and facilitators to the implementation of lay health worker programmes for maternal and child health.
Norwegian branch of the Nordic Cochrane Centre [Internet]. 2012; Disponible en: http://www.kit.nl/ kit/development/DEVDocuments/DEV-DocumentsGlenton-Colvin-2012-Barriers-and-facilitators-toLHWprogrammes-Systematic-Review.pdf.

39. Glenton C, Colvin CJ, Carlsen B, Swartz A, Lewin $\mathrm{S}$, Noyes $\mathrm{J}$, et al. Barriers and facilitators to the implementation of lay health worker programmes to improve access to maternal and child health: qualitative evidence synthesis. Cochrane Database Syst Rev. 2013; 10. 